\title{
Screening auf multiresistente Bakterien - Fluch oder Segen?
}

\author{
Heinz-Michael Just
}

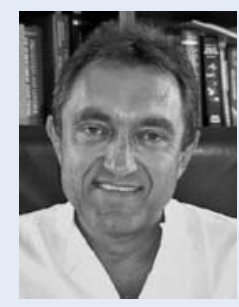

Heinz-Michael Just

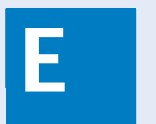

$s$ ist eine allgemeine Erfahrung, dass die Suche nach den Ursachen eines Übels meist zu Erkenntnissen führt, die man eigentlich nicht wollte und die einen belasten. So scheint es auch zunehmend im Bereich des Screenings auf multiresistente Bakterienarten zu sein. Im 10-Punkte-Plan von Bundesgesundheitsminister Gröhe ist der Ruf nach Screening wie ein roter Faden erkennbar. „Alle Krankenhäuser sind verpflichtet, Risikopatienten bei Aufnahme ins Krankenhaus auf multiresistente Erreger zu untersuchen und bis zum Ausschluss einer Besiedelung zu isolieren." Als Begründung werden die KRINKOEmpfehlungen angeführt. Dort steht das aber in dieser Form gar nicht! Die KRINKO empfiehlt stattdessen, Risikopatienten vor multiresistenten Erregern zu schützen. Hierfür kann das Screening ein ebenso probates Mittel sein wie z.B. die Isolierung. Aber eben nicht in jedem Fall, sondern basierend auf einer einrichtungsspezifischen bzw. patientenspezifischen Risikoanalyse (KRINKO-Empfehlung zu MRSA). Zudem muss jeder Patient zum Screening seine Einwilligung geben.

Bei den inzwischen viel bedeutsamer gewordenen multiresistenten gramnegativen Bakterienarten empfiehlt die KRINKO ebenfalls, nur bei definierten Risikopatienten zu screenen und nur auf die „Totalresistenz“ 4MRGN. Herr Gröhe schreibt, „der Bund hat bereits die gesetzliche Grundlage geschaffen, um risikobasierte Screenings auf multiresistente gramnegative Erreger (4 MRGN) schon vor der Aufnahme in ein Krankenhaus erproben zu können“. Seine Vorstellung sei, so schreibt er weiter, dieses Screening „für alle Krankenhäuser verbindlich werden zu lassen“. Da stellen sich einem Krankenhaushygieniker und infektiologisch ausgerichteten Mikrobiologen doch einige Fragen: Warum schafft man erst eine gesetzliche Grundlage und erprobt danach? Eine gut konzipierte klinische Studie zu fördern wäre der sinnvollere Weg gewesen. Warum soll plötzlich etwas „für alle Krankenhäuser verbindlich" werden, wenn das vom eigenen Haus berufene Expertengremium eine risikoadaptierte Vorgehensweise nach wissenschaftlicher Prüfung für sinnvoller hält? Und die entscheidende Frage am Ende der Über- legungskette ist, wer zieht aus den Daten welche Erkenntnisse und was bewirken diese? Ist das Vorgehen wirklich geeignet, das Problem in den Griff zu bekommen, oder schafft es mehr neue und vielleicht größere Probleme?

Im vierten Gröhe-Gebot sollen dann die Meldepflichten verschärft werden, damit die „gefährlichen resistenten Erreger, wie z.B. multiresistente gramnegative Erreger (4MRGN) (...) künftig bereits beim ersten Nachweis des Erregers gemeldet werden“. Und dann? Das Robert Koch-Institut bekommt „notwendige epidemiologische Daten“. Wofür? Was kann das Robert Koch-Institut unternehmen, um die Weiterverbreitung oder - was noch viel wichtiger wäre - die Entstehung zu verhindern? Die Vergangenheit lehrt: zumindest bezogen auf die Ursachenbekämpfung - nichts! Die Ursache der Entstehung liegt doch ganz woanders, nämlich in der Verabreichung von Antibiotika in der Human- und Tiermedizin. Darauf hat das Robert KochInstitut (bislang zumindest) keinen Einfluss - künftig vielleicht über die Empfehlungen der Kommission „Antiinfektiva, Resistenz und Therapie“ (ART), aber nur in der Humanmedizin. Die gramnegativen multiresistenten Bakterien stammen jedoch überwiegend aus der Tiermast, d.h. aus dem Einflussbereich der Veterinärmedizin.

Welche Hilfestellung erwartet sich der Gesetzgeber von den regionalen Gesundheitsämtern für eine medizinische Einrichtung, wenn diese multiresistente Erreger gemeldet hat? Kein Gesundheitsamt kann für eine medizinische Einrichtung mehr Ärzte, mehr Pflegekräfte oder Einzelzimmer aus dem Hut zaubern - dies wären aber die erforderlichen Gegenmaßnahmen!

Das einzig erkennbare Ergebnis bislang ist eine Verunsicherung bei Ärzten, Pflegekräften und auch Patienten, Panikmache durch die Medien in der Bevölkerung und, daraus resultierend, teilweise völlig irrationale Verhaltensweisen. In diesem Heft zeigt Frau Dr. Julia Gräf am Beispiel dreier Urteile deutscher Gerichte sehr schön auf, welche negativen und weitreichenden Folgen diese 
über die Jahre entstandene Meinung, multiresistente Bakterien seien äußerst gefährlich, haben kann (S.155 ff.). Es ist diese unsägliche Kette von Fehlinterpretation, Begriffsverwechslungen, Halbwissen und voreiligen Schlussfolgerungen, die inzwischen eine Situation geschaffen hat, in der eine Besorgnis über die Auswirkungen des Handelns eher angebracht erscheint, als die Besorgnis vor den Erregern selbst.

So geht aus den Urteilen hervor, dass der Nachweis eines antibiotikaresistenten Bakteriums von den Gerichten bereits als Gefährdung eingestuft wird. Stillschweigend wird zwar unterstellt, dass nur an „MRSA“ bzw. die sogenannten „MRGN-Bakterien“ gedacht sei, aber das ist nirgendwo so festgehalten und damit jederzeit erweiterbar. Konsequent wird auch der Nachweis eines solchen Erregers gleichgesetzt mit einer Infektionskrankheit und dies wiederum gleichgesetzt mit einem erhöhten Risiko für eine Übertragung im Sinne einer Ansteckung. Diese Kausalkette ist schlichtweg falsch, weil der Nachweis eines Bakteriums nicht automatisch eine Infektion bedeutet und man gerade bei diesen multiresistenten Bakterien gefunden hat, dass sie häufig nur als Besiedler ohne Krankheitswert vorkommen. Und grundsätzlich pathogener bzw. infektiöser als antibiotikaempfindliche Stämme sind die antibiotikaresistenten nach bisherigen Erkenntnissen auch nicht.

Der nächste Irrtum, der sich durch diese Urteile zieht, ist der, dass Patienten, die mit multiresistenten Bakterienarten besiedelt sind, bei einem vom Arzt verordneten Transport immer einer besonderen medizinischen Betreuung bedürfen, was nur durch einen genehmigten Krankenkraftwagen erfüllt werden könne.
Diese Menschen fahren aber in der weit überwiegenden Mehrzahl alleine Bus und U-Bahn oder nehmen am täglichen Leben teil, ohne dass sie irgendeine Betreuung benötigen. Dabei haben sie einen viel engeren und intensiveren Kontakt zu anderen Menschen, als wenn sie allein in einem Taxi sitzen und nach ihnen eine andere Person in dasselbe Taxi steigt. Die Gerichte ziehen in ihrer Begründung zwei Gesetze heran (das Infektionsschutzgesetz und das Rettungsdienstgesetz Nordrhein-Westfalen), interpretieren diese völlig falsch und machen sich anscheinend über die Tragweite ihrer Auffassung keine Gedanken. Zum einen werden hier gesunde, im Sinn des Allgemeinwohls „ungefährliche“ Personen stigmatisiert. Denn wären sie nach dem von den Gerichten beanspruchten Gesetz „allgemeingefährlich“, dann müssten sie - analog der EinzelzimmerIsolierung in einem Krankenhaus - zum Schutz der Allgemeinheit weggesperrt werden, was bislang - Gott sei Dank! - (noch?) nicht der Fall ist. Zum anderen sind die Leidtragenden die durch die Urteile möglicherweise in ihrer Existenz bedrohten Transportunternehmen. Denen hilft diese Erkenntnis erst, wenn diese widersinnigen, ungerechtfertigten Urteile revidiert worden sind.

Zum Schluss möchte ich noch eine Erweiterung des Herausgeberkreises bekanntgeben und freue mich, dass wir mit Frau Prof. Dr. S. Scheithauer, Herrn Prof. Dr. S. Lemmen und Herrn PD Dr. S. Schulz-Stübner drei neue, unsere Zeitschrift unterstützende Kollegen gewinnen konnten. Der Verlag wie auch die „alten“ Herausgeber freuen sich auf die Zusammenarbeit und erhoffen sich für Zeitschrift und die Leserinnen und Leser zusätzliche neue Impulse. 